\title{
Importancia de las competencias genéricas en la formación del abogado, una apreciación de docentes
}

\section{Importance of generic competences in the training of the lawyer, an appreciation of teachers}

Adriana Romero Sandoval

Universidad Internacional del Ecuador

María Gabriela León

Universidad Andina Simón Bolivar, Ecuador

Marcelo Nicolás Fernandez Schatzer

University of Southern California, EEUU

Autor para correspondencia: adromero@uide.edu.ec; mgabriela_leon@hotmail.com;

marcelof@usc.edu

Fecha de recepción: 18 de julio de 2018 Fecha de aceptación: 15 noviembre del 2018

Resumen: La entrada de las competencias en la educación superior, da cuenta de la necesidad de conectar el mundo educativo con el mundo laboral. Es necesario conocer qué competencias genéricas son importantes en la formación del abogado, desde la percepción de los docentes como actores del proceso enseñanza aprendizaje. El estudio de tipo cuantitativo responde a un diseño descriptivo del estado de la formación de competencias en los estudiantes de Derecho. La investigación de corte transversal recolectó datos en un solo momento, su propósito fue analizar la incidencia de la importancia de las competencias genéricas en una población. Existe coincidencia en el orden de importancia en dos competencias de las consideradas como más importantes para los docentes de Derecho con el proyecto Tuning Latinoamérica: Capacidad de abstracción, análisis y síntesis y Capacidad para identificar, plantear y resolver problemas. Mientras que con el proyecto Tuning Europa, se presentan también dos coincidencias: Conocimientos generales básicos y Capacidad crítica y autocrítica. Las normas ecuatorianas demandan un cambio en la agenda de la docencia universitaria, sin embargo, una educación por competencias con docentes de Derecho alejados del libre ejercicio profesional por la carga laboral pone en duda el desarrollo de competencias en los estudiantes universitarios.

Palabras claves: educación superior; competencias genéricas; abogado; docente

Abstract: Higher education needs to be linked to the needs of practice of professionals. It is necessary to understand which generic skills are important in the formation of legal practitioners from a faculty perspective. This quantitative analysis builds up on a descriptive study of the current state of competency building of law students. Data was collected at once in a cross sectional study, providing a momentary snapshot. The purpose was to analyze evidence of the importance of generic skills in a group. There is coincidence between the competencies that law faculty consider as important with the ALFA Tuning Latin America project: Capacity to abstract, analysing and synthesizing as well as problem identification, formulation and solution capacities. There are two coincidences with the Tuning Europe project: Basic general knowledge and critical and autocritical capacity. Policy Ecuadorian reform has forced law faculty to take on more administrative and 
research responsibility, driving them away from practicing the law profession. This casts doubt that students will be able to develop the necessary practical capabilities of legal practitioners.

Key words: higher education; generic skills; lawyer; faculty

\section{Introducción}

En la segunda década del siglo XXI, la educación superior en América Latina presenta desafíos vinculados a políticas públicas para aumentar el acceso y la permanencia de estudiantes, a la calidad de la formación profesional y a la pertinencia social y profesional de las instituciones de educación superior y su oferta académica. Estos desafíos han supuesto reflexiones acerca de la educación en el campo del Derecho.

En Ecuador, la educación superior es considerada un área estratégica, parte de un sistema que contribuye a la sociedad, a la estructura social, productiva y ambiental; formando profesionales con capacidades y conocimientos que respondan a la necesidad de conectar el mundo educativo con el mundo laboral. La valoración mediante estudios sistemáticos sobre la respuesta que da la educación superior a las necesidades de la sociedad deja aun la tarea pendiente de la pertinencia (Jiménez-Vivas, 2009).

Uno de los estudios que identifica y atiende las necesidades de la sociedad es el Proyecto Tuning en América Latina para el área del Derecho, estudio en el que participan una red de comunidades de académicos que reflexionan y debaten sobre esta disciplina. Ello significa que también los docentes deben vivir las relaciones pedagógicas de forma activa, reflejándolas permanentemente en sus prácticas y actitudes como educadores (Beneitone, Esquetini, González, Maletá, Siufi, Wagenaar, 2010).

La entrada de las competencias en la educación superior da cuenta de la imperiosa necesidad de conectar el mundo educativo con el mundo laboral, por lo que es necesario conocer qué competencias genéricas son importantes en la formación del abogado, desde la percepción de los docentes como actores del proceso enseñanza aprendizaje.

\section{La educación superior del siglo XXI}

Las reflexiones sobre la educación para el siglo XXI, se atribuyen, entre otros autores, a la Organización de las Naciones Unidas para la Educación, la Ciencia y la Cultura, por sus siglas en inglés UNESCO, que prescribe a la educación como un instrumento indispensable para el progreso de la humanidad en camino hacia la paz, la libertad y la justicia social; con una función esencial que es el desarrollo continuo de la persona y la sociedad (UNESCO, 2017).

La educación superior siglo XXI se presenta con una fisonomía de cambio, que conlleva características como: la masificación en la matriculación, el aprendizaje a distancia, la educación para toda la vida, la tensión entre lo mundial y lo local, el dilema de la mundialización de la cultura, las nuevas tecnologías de la información, la concentración en los problemas inmediatos, la competencia y la igualdad de oportunidades, el desarrollo del conocimiento y la capacidad de asimilación del ser humano, el respeto al pluralismo, la superación del sí mismo y la incertidumbre valorativa (UNESCO, 2016). 
Del mismo modo, la educación superior se enfrenta a desafíos y dificultades relativos al financiamiento, a la igualdad de condiciones en el acceso a los estudios y permanencia en ellos, mejor perfil del docente, la formación basada en competencias, mejora y calidad en la enseñanza, investigación y consultoría, pertinencia de los programas de estudio, la internacionalización de la universidad, el uso de la tecnología como parte de la metodología didáctica en aula, y demás características que responden al cambio como una constante de momento (Cid, Cuadra, Cuevas, \& Villalobos, 2017).

Los modelos sociales, políticos y económicos que han marcado el pasado inmediato son insuficientes, y en muchos casos obsoletos e inadecuados para entender y actuar sobre el mundo actual. Los cambios sociales, culturales, económicos, presentes en el siglo XXI demandan una permanente reconfiguración que incide en los planteamientos educativos, para (Tejada Fernández, 2002) la educación “exige adaptarse cultural, social, laboral, profesional y personalmente al ritmo del cambio y su velocidad, cifrado en nuevas claves de concepciones culturales, de producción, de relaciones sociales, económicas e industriales" (Tejada, 2002, p. $30)$.

Los nuevos alumnos presentan características de una cultura propia construida a partir de nuevos conocimientos y nuevos valores, además de hijos son padres, son solteros, casados, son jóvenes, adultos, combinan estudio con trabajo, la diversidad en cuanto a edad, origen social, económico, cultural, geográfico, trayectoria formativa, experiencia laboral, necesidades especiales donde la palabra ha perdido valor; todo está cambiando hacia una mayor semejanza con la estructura social de la propia sociedad. Pero también se presenta una nueva realidad de deserción, abandono y repitencia (Rama Vitale, 2006).

El papel de la universidad en el contexto del siglo XXI, al incorporar la tecnología a la oferta formativa, posibilita el incremento de nuevos servicios como la educación a distancia o modalidad semipresencial con las ventajas que estos modelos formativos conllevan. Por otro lado, la etapa universitaria deja de ser una meta en el proceso formativo y se convierte en una etapa más en la educación a lo largo de toda la vida. En respuesta a la globalización, la universidad recupera y amplía las diversas formas de internacionalización de larga duración, participando en un mundo polarizado entre globalizaciones contradictorias (Sousa Santos, 2005).

La formación basada en competencias surge como una respuesta al desajuste entre la formación y el empleo, está caracterizado por varios criterios que describen y evalúan los programas basados en competencias, se presenta como una alternativa a los enfoques tradicionales de la formación basada en el conocimiento y para acercar los objetivos de formación a las exigencias del empleo expresadas en términos de competencias profesionales (Blas Aritio, 2014).

Consiste en un enfoque distinto, no opuesto ni excluyente, donde la formación es un instrumento para alcanzar o conseguir algo. Dicho de otra manera, adquirir las competencias profesionales requeridas en el empleo. Los enfoques tradicionales y el enfoque por competencias continúan compartiendo procedimientos en el diseño de los programas de formación (Tejada Fernández, 2012). 
La formación basada en competencias tiene carácter holístico, busca integrar las experiencias de la vida real, con el propósito de desarrollar habilidades mediante tareas y funciones básicas donde se domine los conocimientos, habilidades, actitudes y valores que determinan el logro de la competencia (Argudín, 2009).

La formación basada en competencias es un fenómeno que revela para Rodríguez \& Parietti (2007) la relación que se establece entre Estado y mercado laboral, donde la educación afirma el rol del Estado en favor del mercado laboral y con dependencia del vínculo educacióntrabajo que valora y valida los conocimientos convertidos en competencias, donde el sector productivo y el sector educativo, por si solos, no han podido encontrar un equilibrio y desarrollo constante (Rodríguez \& Parietti, 2007).(Rodríguez \& Parietti, 2007)

El mercado laboral, cuando se ocupa de validar los saberes, lo hace según su lógica, es decir, desecha fundamentos conceptuales y reflexiones sobre el sentido de las acciones humanas. La validación es inmediatista y rentable, asociada a maximizar la utilidad; sin embargo, en la última década cuando la economía social y solidaria ha tomado protagonismo en América Latina, es necesario reconocer otras formas de economía que permita a la educación cubrir estos modos de producción y reproducción (Coraggio, 2005).

La reconfiguración de la educación tiene que ver con la velocidad del conocimiento que deja de ser lento, estable y escaso; presentándose continuo y en constante expansión y renovación. En la medida en que la formación opera con el conocimiento se plantea: ¿qué contenidos seleccionar?, ¿con qué criterios y cómo manejar la renovación o integración de lo nuevo? El texto escrito y la palabra del docente no es el único medio de información y el perfil de los alumnos ha cambiado (Mas Torelló \& Tejada Fernández, 2013).

\section{Competencias genéricas}

La palabra competencia para el presente estudio, hace referencia a saber los conocimientos requeridos por la profesión, donde el ejercicio eficiente y efectivo de los conocimientos requiere un saber hacer; y para responder a un mundo cambiante se necesita saber estar y saber ser. En este concepto es determinante los dos primeros saberes que responden a la profesión, saber y saber hacer; y los dos últimos saberes, saber estar y saber ser, corresponde a las competencias genéricas (López Gómez, 2016).

La competencia para Mulder (2007), tiene dos significados esenciales: (a) Autoridad: exteriorizar la responsabilidad, la autorización o el derecho a decidir, producir, prestar un servicio, actuar, ejercer o reclamar. (b) Capacidad: poseer los conocimientos, las aptitudes y la experiencia para ejercer. La capacidad de utilizar conocimientos y habilidades, de manera transversal e interactiva, en contextos y situaciones que requieran la intervención de conocimientos vinculados a diferentes saberes (Mulder, Weigel, \& Collins, 2007).

Las capacidades que se clasifican en cognitivas, psicomotrices y afectivas, son el aspecto nuclear de las competencias, porque sin la dinamización de las capacidades es imposible llegar a ser competente, esto implica dinamizar recursos como: capacidades materiales, conocimientos, 
saberes, saber hacer, recursos externos y recursos internos. La competencia exige un saber entrar en acción, una puesta en juego, un orden para resolver cosas, enfrentarse a tareas complejas, en situaciones o tareas variadas y definidas en un campo disciplinario determinado; con característica de dominio, habilidad adquirida, actuación eficaz haciendo frente a las labores (Fernández, González, \& Román, 2016) .

La competencia para Mas Torelló (2011) va más allá que una acumulación de contenidos (saber), está constituida por habilidades (saber hacer), actitudes y valores (saber ser y estar), que se desarrollan mediante la propia experiencia socio laboral. La competencia se relaciona con la capacidad de utilizar conocimientos y habilidades, de manera transversal e interactiva, en contextos y situaciones que requieran la intervención de conocimientos vinculados a diferentes saberes, lo que implica la comprensión, reflexión y discernimiento teniendo en cuenta la dimensión social de cada situación (Mas Torelló, 2011).

Al incluir la palabra profesional o laboral al término competencia, se le asocia al concepto de capacidades (el saber hacer) y a los conocimientos (el saber), por ser atributos personales que se movilizan en el momento de ejercer una competencia profesional donde tienen lugar los procesos productivos reales o simulados; es la realización efectiva de los roles y situaciones de trabajo relacionadas con los resultados de las acciones o actividades de trabajo (Blas Aritio, 2014).

La competencia profesional para Tejada (2009) es mucho más que una acumulación de contenidos, saberes; también está constituida por habilidades, saber hacer; actitudes y valores, saber ser y estar; demostrando la capacidad del docente al activar todos estos saberes en el contexto laboral en la solución de situaciones propias de su rol de docente (Tejada Fernández, 2009).

Las competencias responden a una serie de tipologías que, para utilidad de este estudio, tomará la clasificación propuesta por varios autores y expertos, esto es, aquella que la agrupa en dos grandes bloques, las competencias genéricas y las específicas. Las competencias genéricas son aquellas que facilitan las herramientas básicas que necesita el profesional para analizar las situaciones, problemas, manejar estrategias y aportar soluciones adecuadas, aquellas necesarias para el empleo y para el desarrollo de la ciudadanía responsable, se aplican a todos los campos ocupacionales, llamadas también transversales. Las competencias específicas, por su parte, hacen referencia al conocimiento teórico y metodológico de cada profesión, en este caso el campo de la jurisprudencia, demostrando una cualificación profesional concreta del docente de Derecho (Mas Torelló \& Tejada Fernández, 2013).

El proyecto Tuning, una de las investigaciones sobre competencias en la educación superior, distingue tres tipos de competencias genéricas, las instrumentales que hacen referencia a las capacidades cognitivas, metodológicas, tecnológicas y lingüísticas; las competencias interpersonales que son las habilidades sociales de interacción y cooperación; y las competencias sistémicas que son capacidades y habilidades relacionadas con la combinación de comprensión, sensibilidad y conocimientos (González \& Wagenaar, 2006). 
La competencia del factor humano se reconoce al interior de una organización para alcanzar objetivos, el grado de competencia de un trabajador para acceder a un trabajo se hace cada vez mayor donde la demanda de habilidades y conocimientos es necesaria para poder desempeñarse en un puesto de trabajo. Los desafíos de la globalización frente al mercado laboral hacen que el perfil del trabajador vaya cambiando a ser más autónomo, más cualificado, con mayor flexibilidad, creatividad e iniciativa, con mejor capacidad de adaptación, de innovación, de gestión del cambio, de trabajo en equipo, de resolución de problemas (Mas Torelló \& Tejada Fernández, 2013).

Las competencias genéricas en la formación del Derecho propician un aprendizaje pertinente y pueden asegurar posibilidades reales de una inserción laboral en áreas diversificadas. Considerando que los problemas jurídicos pueden ser abordados desde otras áreas de conocimiento, estas competencias permiten un diálogo epistemológico y metodológico con otros campos (Musse et al., 2014).

El proyecto Tuning América Latina, reflexiones y perspectivas en Derecho, según Musse et al. (2014) identifica 15 competencias genéricas que se deben fomentar en los estudiantes de educación superior, como se detalla a continuación:

1. Capacidad de identificar, plantear y resolver problemas aplicando los conocimientos.

2. Capacidad de organización y planificación.

3. Responsabilidad social y compromiso ciudadano.

4. Capacidad de comunicar los conocimientos disciplinarios en distintos contextos

5. Capacidad de investigar, mediante la búsqueda, el procesamiento y el análisis de la información procedente de fuentes diversas.

6. Capacidad de aprender y actualizarse permanentemente.

7. Capacidad crítica y de autocrítica.

8. Capacidad para actuar en nuevas situaciones de forma creativa.

9. Capacidad de tomar decisiones justificadas.

10. Capacidad de trabajar en equipo, motivándolo y conduciéndolo a metas comunes.

11. Habilidades interpersonales.

12. Compromiso con la preservación del medio ambiente.

13. Valoración y respeto por la diversidad y por la multiculturalidad. 


\section{Compromiso ético.}

\section{Compromiso con la calidad.}

\section{Docente universitario}

El nuevo rol del docente está influenciado por la incorporación de la tecnología en la universidad, al contar con clases y campos virtuales, bibliotecas digitales, comunicación electrónica, manejo de sistema documentales, pizarras inteligentes y demás medios que se han puesto al servicio del docente para llevar a cabo su actividad cotidiana; exige de éste el uso permanente y dominio de la tecnología. De la misma manera, cuando el estudiante tiene acceso a la información a través del internet, el rol del docente deja de ser transmisor de información y da apertura a un rol de orientación, apoyo, guía, dinamizador en una situación de aprendizaje (Mas Torelló \& Tejada Fernández, 2013).

Para Tejada (2009) el término docente se refiere a toda persona que tiene relación con la formación desde la óptica de la responsabilidad en la gestión, la concepción de la formación y la realización de la misma. Detrás del término docentes se encuentra una variedad de profesiones que actúan en diferentes contextos y con diversos recorridos formativos (Tejada Fernández, 2009).

Las numerosas reformas de planes de estudio suponen un cambio de planteamientos didácticos en varias disciplinas, el desarrollo de una clase gira en torno a la selección crítica de materiales, recursos, fuentes de información que se encuentren en bases de datos electrónicas, campus virtuales, experiencias de conocimientos que se encuentren en internet; además, la hetorogeneidad de los alumnos hace necesario que los docentes trabajen en equipo, condición que no se había considerado porque el trabajo docente tiene la característica de ser individual y tener como referente el aula de clase.

La necesidad de revalorizar la función docente del sistema de educación superior ha merecido varios estudios y debates, al respecto, Aucejo (2009) en el análisis de la enseñanza del Derecho para el siglo XXI, hacer referencia a la condición que debe tener el docente frente a un sistema de calidad de educación superior, este manifiesto a cargo de Standards and guidelines for Quality Assurance in the European Higher Education Area (ENQA) "condiciona el sistema de calidad a que los docentes tengan un conocimiento y comprensión completos de la materia que enseñan y dispongan de las habilidades y experiencias para transmitir sus conocimientos" (Aucejo, 2009, p.18). (Andrés Aucejo, 2009)

El personal académico de una institución de educación superior está conformado por los docentes o profesores e investigadores titulares y no titulares. La condición de titularidad se adquiere al ingresar a la carrera y escalafón del profesor e investigador a través de un concurso público de méritos y oposición. Formar parte de la carrera docente asegura estabilidad laboral. El personal académico puede cumplir las actividades de docencia, investigación y de dirección o gestión académica distribuidas en función del tiempo de dedicación de cada docente, que puede ser: tiempo completo, medio tiempo o tiempo parcial (CES, 2017). 
Las actividades, funciones y competencias que debe tener un docente universitario no son siempre las mismas, son dinámicas, se van definiendo con base en el cambio y demanda que tiene la institución de educación superior, el sistema de educación superior, la política que rige el sistema de educación, la tecnología y las exigencias de la sociedad en su conjunto; estas funciones son desarrolladas y toman relevancia en el marco de tres escenarios donde el docente interactúa: (1) Un contexto general, que hace referencia al entorno social, profesional, laboral y cultural; (2) Un contexto institucional, relacionado a la universidad, facultad, escuela, y; (3) Un micro contexto, es decir, el aula, laboratorio, oficina, auditorio (Mas Torelló \& Tejada Fernández, 2013).

Las actividades del docente, denominado también como personal académico, se encuentran establecidas y delineadas, en el caso ecuatoriano, en el Reglamento de Carrera y Escalafón del Profesor e Investigador del Sistema de Educación Superior. La primera actividad, la docencia, que es la actividad asociada al conocimiento y aprendizaje, demanda del profesor incitar el interés por aprender, cómo aprender y mantener al día estos conocimientos; para alcanzar estas obligaciones se necesita contar con capacidades, conocimientos, habilidades, actitudes y valores (Díaz, 2017).

El papel del docente como reproductor de conocimiento ha sido reemplazado por un orientador de los aprendizajes, de este modo, son los estudiantes quienes deben saber buscar, procesar y utilizar el nuevo contenido aprendido, en función del conocimiento previo. Esto demanda una concepción del proceso de aprendizaje que provea la adquisición de las capacidades, identifique estrategias comunes para solucionar problemas y desarrolle capacidades socio- afectivas como valores, actitudes, motivaciones y emociones. El rol del profesor no se ve limitado a la adquisición de conocimientos y al desarrollo de destrezas, sino que también tiene una gran importancia el desarrollo de los valores (Mas-Torelló \& Olmos-Rueda, 2016).

La segunda actividad, la investigación, ha sido objeto de cambios en virtud de la reforma universitaria implementada por los órganos estatales en Ecuador en la última década, reformas que han dado como resultado una estrecha combinación entre la enseñanza y la investigación; hoy se fomenta la investigación competitiva, la creación de equipos multidisciplinarios formados por miembros de universidades de diversos países, donde el desarrollo de investigación debe estar asociado un proceso de planificación sostenible y que responde a líneas y grupos de investigación que demuestren un impacto en la sociedad (Armijos, Barreno, \& Sánchez, 2015).

Es necesario mencionar que en la oferta académica existen carreras cuya formación profesional está ligada a la producción de conocimiento científico, y otras en las cuales la investigación no posee la misma fuerza, es decir, las llamadas carreras profesionalizantes. Esta dicotomía genera un debate acerca de la función de la universidad y del tipo de investigación que cada unidad académica puede y debe realizar.

Para producir conocimiento científico hay que producir científicos, por lo que queda la inquietud acerca de la capacidad de dudar en las generaciones de alumnos que se recibe en el aula. El marco normativo supone a un alumno universitario con potencial científico que dejó de ser un sujeto pasivo del aprendizaje y ha pasado a ser un sujeto activo capaz de aumentar el 
conocimiento o refutar el disponible. El docente que trabaja con este alumno debe estar apto para llevar a cabo el desarrollo de la investigación y la adquisición del perfil profesional que la universidad busca formar (Espinosa \& Aguilar, 2017).

El cambio en la capacidad investigativa de la universidad se debe en buena parte a la inclusión de la tecnología y acceso a las tecnologías de la información y comunicación, que facilita el acceso a bibliotecas virtuales, bases de datos, documentos y demás fuentes de información; la capacidad de manejo y procesamiento de bases de datos, la comunicación al momento del conocimiento científico, el intercambio de conocimiento con otros investigadores en todo el mundo a través de correo, videoconferencia, mensajería; la capacidad de almacenamiento de gran cantidad de información en medios portátiles o en entornos virtuales denominados la nube y el interés de organizaciones por proponer y financiar la investigación en temáticas específicas (Zambrano, 2017) .

La tercera actividad del docente es la gestión. El profesor como parte integrante de una institución, puede participar en tareas de su organización, pero no como un compromiso personal adquirido con la institución donde labora, procurando que esto no afecte a las actividades de docencia; por el contrario, las tareas administrativas están asociadas a la adaptación de las necesidades e intereses de la institución, facultad y escuela, donde es preciso el manejo de idiomas, el uso de la tecnología, la capacidad comunicativa, la organización del trabajo, la habilidad de alcanzar logros, el manejo de recursos económicos, adaptación al cambio, seguimiento y ejecución de presupuesto (Romero-Sandoval \& Leon Guajardo, 2017).

La cuarta actividad, la vinculación con la comunidad, desde la visión del marco normativo ecuatoriano, demanda del docente un conocimiento en el manejo de proyectos, el uso de recursos humanos, físicos y económicos, el uso efectivo del tiempo, la identificación de una línea base que en su momento permita definir los indicadores de impacto del proyecto; dominio de planificación para que las actividades que desarrolla el estudiante participante del proyecto de vinculación, tenga perfecta coherencia con la formación que está recibiendo en la carrera, manejo de documentos, elaboración de reportes, capacidad comunicativa con los organismos de control que vigilan el trabajo de vinculación para la carrera de Derecho. La legislación que regula la vinculación con la comunidad en la carrera de Derecho, afecta y determina la organización del trabajo, funciones y competencias del docente (Registro Oficial, 2010).

La capacitación y actualización científica es otro de los elementos considerados para el ascenso de categoría dentro del escalafón. En el caso de las instituciones públicas, estas deben elaborar un plan de capacitación y perfeccionamiento docente para cada período académico, tomando en cuenta las necesidades del personal académico y los objetivos institucionales. El Reglamento General a la Ley Orgánica de Educación Superior (2011) establece que, con el fin de garantizar el acceso a la formación y capacitación de los profesores e investigadores, todas las instituciones de educación superior establecerán en su presupuesto, al menos el uno por ciento para el cumplimiento de estas actividades (Presidencia de la República, 2011).

Los programas y actividades de capacitación docente también son considerados en los diferentes procesos de evaluación externa y acreditación de las instituciones de educación superior, así como de sus carreras y programas. Una vez que el personal académico forma parte 
de las universidades y escuelas politécnicas, adquiere a su una serie de derechos, encabezados por el de libertad de cátedra que, según la Ley Orgánica de Educación Superior (2010) radica en el ejercicio de la cátedra o investigación sin ningún tipo de presiones de índole religiosa, partidista o política. Del mismo modo, los docentes e investigadores tienen el derecho a participar en el proceso de construcción, difusión y aplicación del conocimiento, teniendo a disposición el acceso a condiciones necesarias para el ejercicio de estas actividades. Los docentes también derecho a recibir capacitación periódica orientada a su formación profesional y a la cátedra que imparten, lo que contribuirá al desarrollo personal, profesional y académico (Registro Oficial, 2010).

Los demás derechos garantizados por la ley a los docentes dicen relación con el acceso a la carrera de profesor e investigador que garantice estabilidad, promoción, movilidad y retiro, teniendo como base los méritos académicos, calidad de la enseñanza, capacitación permanente y los resultados de investigación. De igual manera, pueden acceder a cargos directivos, así como también pueden ser elegidos como representantes de los docentes al cogobierno o máximo órgano colegiado de las universidades y escuelas politécnicas, sin ningún tipo de discriminación. Del mismo modo, tienen derecho a participar en el proceso de evaluación institucional (Registro Oficial, 2010).

El perfil profesional del docente está conformado por diversos aspectos como: (1) Características personales que debe tener de manera particular, esto es el rasgo de personalidad; (2) Habilidades y destrezas que deben caracterizar su quehacer y que se reflejan en las funciones a desempeñar por el docente; (3) Conocimientos y actitudes necesarias para el ejercicio de la docencia; (4) Acciones generales y específicas que desarrolla el docente en su contexto para la solución de las necesidades sociales identificadas (Romero-Sandoval \& Leon Guajardo, 2017).

En términos generales se puede decir que el docente universitario, en este caso, el de Derecho, debe planificar, compartir, guiar y evaluar acciones formativas, utilizando y elaborando recursos didácticos, bajo criterios de calidad del proceso de enseñanza aprendizaje.

\section{El perfil del Abogado}

El mercado laboral se va transformando de acuerdo a los cambios de las fuerzas productivas que responden a las exigencias de la era que vivimos, la profesión del abogado que goza de interés y prestigio social, se enfrenta a los grandes desafíos de los cambios en la estructura del mundo del trabajo. En los últimos años se han presentado limitaciones en cuanto a la capacidad con que los egresados ejercen sus profesiones, poniendo al mercado laboral en una expectativa de contratación limitada y un nivel de insatisfacción en la solución de problemas profesionales, donde la presencia de características asociadas a las nuevas tecnologías, actores diversos con roles diversos, producción a escala mundial y ciclos cortos de los productos; marcan la desigualdad de profesionales egresados de las universidades (Delgado, García, \& Meza, 2010).

La necesidad de un trabajador más cualificado y autónomo, con mayor flexibilidad, iniciativa y creatividad, con capacidad de adaptación, innovación, gestión del cambio, solución de problemas, presenta nuevas tendencias de organización social que acompañan al 
planteamiento de una educación que centralice en la persona el protagonismo de su toma de decisiones y de sus proyectos personales, donde el sujeto tiene un rol innovador cuando sostiene y desarrolla sus propias competencias y reconstruye proyectos de vida (Sánchez Torelló, 2014).

En el ámbito del Derecho para el siglo XXI, la palabra continúa siendo la herramienta básica del jurista, sin embargo, y según de Prada (2015), el lenguaje jurídico sigue siendo, hoy por hoy, ajeno al de los ciudadanos a los que consecuentemente, aún hoy se considera, con mínimas excepciones, incapaces de defenderse por sí mismos civil o penalmente. Esta lejanía del lenguaje jurídico con el lenguaje común tiene, entre otras causas, la ausencia de contenidos jurídicos en la formación básica de los ciudadanos, cuestión que hace difícil el manejo de textos jurídicos como contratos, declaraciones de impuestos, actas, citaciones, sentencias, entre otros; lo que revela el desconocimiento de términos jurídicos que, cuyo manejo aparentemente debiera resultar habitual. Por otro lado, los aspectos lingüísticos como la redacción, manejo de estilos propios del lenguaje jurídico, en si la calidad verbal y escrita resulta fundamental para el trabajo de un abogado ya que no puede concebir que no le entiendan o le entiendan mal (Salgado, 2015).

El derecho es una profesión en constante transformación, con una alta dependencia de los modelos jurídicos y las coyunturas económicas y políticas, que debe actualizarse al ritmo de los cambios institucionales que, a su vez, se ven reflejados en permanentes reformas normativas. De hecho, solo en el periodo 2013-2017 el órgano legislativo ecuatoriano aprobó sesenta y nueve leyes, todos cuerpos normativos que introducen nueva regulación o que reforman la ya existente (Asamblea Nacional, 2017). En virtud de ello, la universidad debe ser muy ágil, pero prudente, para dar respuesta al compás de los acontecimientos sociales. El derecho se presenta como una profesión con características asociadas al formalismo, a la gestión de relaciones públicas, al dominio de la ley; dejando de lado los valores necesarios en el ejercicio profesional como: humildad, honestidad, solidaridad y ética (Ramos, 2016).

Tomando en cuenta que es una profesión altamente regulada no sólo por la sociedad, sino a través de normas, vigilancia y sanciones a cargo de los organismos gubernamentales de control y que, específicamente en el caso ecuatoriano, la propia legislación la ha incluido entre aquellas profesiones cuyo ejercicio puede comprometer el interés público (CES, 2013); debe responder, entonces, con una adecuada aplicación de conocimientos y actitudes en el ejercicio de la profesión, considerando el riesgo moral y la competencia laboral, sumado a ello dentro de aquellas profesiones.

La formación del profesional del Derecho está enmarcada para De Vivero (2014) en tres grandes objetivos: (1) Proporcionar al estudiante los conocimientos y la estructura básica del derecho; (2) La adquisición de habilidades prácticas en la aplicación y manejo de conocimientos en las diversas situaciones nuevas que afronta, de tal manera que pueda evidenciar su compresión; y (3) El estudiante debe asumir que en su calidad de abogado tiene un rol notable en la sociedad que le obliga a tomar decisiones dentro de la ética y con alto contenido social (De Vivero, 2014).

\section{Métodos}


El estudio de tipo cuantitativo responde a un diseño descriptivo ya que la investigación de este tipo representa el estado, las características y los factores presentes en aquellos fenómenos y hechos que ocurren en forma natural (Sáez López, 2017). La investigación de corte transversal recolectó datos en un solo momento, su propósito fue describir las variables participantes y analizar la incidencia e interrelación en un momento dado, se trata de una exploración inicial en una situación específica. Con esto se logró indagar la incidencia de la importancia de las competencias genéricas en una población (Hernández Sampieri, Fernández Collado, \& Baptista Lucio, 2014).

El cuestionario utilizado en el estudio corresponde al instrumento de recolección de datos para conocer la importancia de las competencias genéricas, empleado en el Proyecto Tuning América Latina 2004 - 2007, un proyecto que está dado para la búsqueda de puntos comunes de referencia centrado en las competencias. Entre las primeras tareas del proyecto fue encontrar las competencias genéricas para América Latina, por lo que se trabaja con 27 competencias genéricas(Beneitone et al., 2010).

La metodología internacional Tuning permite comparar la información de la carrera de derecho con los resultados de los estudios de competencias genéricas en Latinoamérica realizado en los años 2004 al 2007, donde participaron 18 países con 62 universidades y 4558 académicos. Así mismo, se puede comparar con el resultado del estudio en Europa realizado en los años 2002 al 2004, donde participaron 100 universidades con 988 académicos de 16 países europeos.

El escenario natural del estudio es una universidad particular, autofinanciada con veinte y cinco años de funcionamiento en la capital de Ecuador. El cuestionario fue entregado a los docentes de la carrera de derecho en abril de 2017, participaron 25 docentes. En el cuestionario se presenta una lista de 27 competencias genéricas, tomadas del proyecto Tuning Latinoamérica, donde los docentes tuvieron que organizar las competencias desde la más importante, puntuando con valor menor, a la menos importante, puntuando con valor mayor.

Las competencias genéricas que participan en el estudio son: capacidad de abstracción, análisis y síntesis; capacidad de aplicar los conocimientos en la práctica; capacidad para organizar y planificar el tiempo; conocimientos sobre el área de estudio y la profesión; responsabilidad social y compromiso ciudadano; capacidad de comunicación oral y escrita; capacidad de comunicación en un segundo idioma; habilidades en el uso de las tecnologías de la información y de la comunicación; capacidad de investigación; capacidad de aprender y actualizarse permanentemente; habilidades para buscar, procesar y analizar información procedente de fuentes diversas; capacidad crítica y autocrítica; capacidad para actuar en nuevas situaciones; capacidad creativa; capacidad para identificar, plantear y resolver problemas; capacidad para tomar decisiones; capacidad de trabajo en equipo; habilidades interpersonales; capacidad de motivar y conducir hacia metas comunes; compromiso con la preservación del medio ambiente; compromiso con su medio socio-cultural; valoración y respeto por la diversidad y multiculturalidad; habilidad para trabajar en contextos internacionales; habilidad para trabajar en forma autónoma; capacidad para formular y gestionar proyectos; compromiso ético y compromiso con la calidad (Beneitone et al., 2010). 
La construcción de la base de datos y los análisis se realizó en el paquete estadístico SPSS v24. El enfoque cuantitativo demanda una necesidad de observar ciertas reglas éticas en el uso de la estadística, consideraciones acerca del anonimato y la confidencialidad en el uso de los datos y resultados obtenidos, la no alteración de los datos con los que se trabaja, o se elimine aquellos casos que alejan los resultados obtenidos de lo esperado.

La confidencialidad del participante en la encuesta es asegurada mediante la asignación de un código el cual permitió encriptarlo en todos los análisis y reportes. La observación de los derechos del participante se refleja en el consentimiento informado y la declaración del participante. El protocolo de investigación fue presentado y aprobado por el Comité de Ética de la Universidad Internacional del Ecuador.

\section{Resultados}

Los docentes de la carrera de derecho identifican la importancia de las 27 competencias genéricas como se muestra en la Tabla 1. Los docentes de Derecho que participan en el estudio consideran como las seis competencias más importantes las siguientes: Conocimientos sobre el área de estudio y la profesión, Capacidad de aplicar los conocimientos en la práctica, Capacidad de abstracción, análisis y síntesis, Capacidad de aprender y actualizarse permanentemente, Capacidad para identificar, plantear y resolver problemas, Capacidad crítica y autocrítica.

Tabla 1. Importancia de las competencias genéricas para el perfil de Abogado

\begin{tabular}{ll}
\hline Competencia & Importancia \\
\hline $\begin{array}{l}\text { Conocimientos sobre el área de estudio y la } \\
\text { profesión }\end{array}$ & 1 \\
$\begin{array}{l}\text { Capacidad de aplicar los conocimientos en la } \\
\text { práctica }\end{array}$ & 2 \\
Capacidad de abstracción, análisis y síntesis & 3 \\
$\begin{array}{l}\text { Capacidad de aprender y actualizarse } \\
\text { permanentemente }\end{array}$ & 4 \\
Capacidad para identificar, plantear y resolver & \\
problemas & 5 \\
Capacidad crítica y autocrítica & 6 \\
Compromiso ético & 7 \\
Capacidad para tomar decisiones & 8 \\
Capacidad de comunicación oral y escrita & 9 \\
Capacidad de investigación & 10 \\
Capacidad de trabajo en equipo & 11 \\
Compromiso con la calidad & 12 \\
Capacidad para organizar y planificar el tiempo & 13 \\
Capacidad creativa & 14 \\
Habilidades para buscar, procesar y analizar & \\
información procedente de fuentes diversas & 15 \\
Habilidades interpersonales & 16 \\
Capacidad para formular y gestionar proyectos & 17 \\
Capacidad para actuar en nuevas situaciones & 18 \\
Responsabilidad social y compromiso ciudadano & 19 \\
\hline
\end{tabular}




\begin{tabular}{ll}
\hline Competencia & Importancia \\
\hline Habilidad para trabajar en forma autónoma & 20 \\
Capacidad de comunicación en un segundo idioma & 21 \\
Valoración y respecto por la diversidad y & 22 \\
multiculturalidad & \\
$\begin{array}{l}\text { Capacidad de motivar y conducir hacia metas } \\
\text { comunes }\end{array}$ & 23 \\
Compromiso con su medio socio-cultural & 24 \\
Habilidad de trabajar en contextos internacionales & 25 \\
$\begin{array}{l}\text { Compromiso con la preservación del medio } \\
\text { ambiente }\end{array}$ & 26 \\
Habilidades en el uso de las tecnologías de la \\
información y de la comunicación
\end{tabular}

En el proyecto Tuning Europa, los académicos consideraron como las seis competencias más importantes las siguientes: Conocimientos generales básicos, Capacidad de análisis y síntesis, Capacidad de aprender, Capacidad para generar nuevas ideas (creatividad), Capacidad de aplicar los conocimientos en la práctica y Capacidad crítica y autocrítica.

De acuerdo a los reportes internacionales presentados en el proyecto Tuning Latinoamérica los académicos consideraron como las seis competencias más importantes las siguientes: Compromiso ético, Capacidad de aprender y actualizarse, Capacidad de abstracción, análisis y síntesis, Capacidad de aplicar los conocimientos en la práctica, Capacidad para identificar, plantear y resolver problemas, Compromiso con la calidad.

Se puede apreciar una coincidencia, en el orden de importancia, en dos competencias de las consideradas como más importantes para los Docentes de Derecho con el proyecto Tuning Latinoamérica: Capacidad de abstracción, análisis y síntesis y Capacidad para identificar, plantear y resolver problemas. Mientras que con el proyecto Tuning Europa, se presentan también dos coincidencias: Conocimientos generales básicos y Capacidad crítica y autocrítica.

Con respecto a las 15 competencias identificadas en las reflexiones y perspectivas en Derecho del proyecto Tuning (2014), se puede observar que hay cinco competencias genéricas que los docentes de Derecho participantes del estudio identifican, pero no consta en las 15 competencias del proyecto Tuning:

$\checkmark$ Conocimientos sobre el área de estudio y la profesión

$\checkmark$ Capacidad de aplicar los conocimientos en la práctica

$\checkmark$ Capacidad de abstracción, análisis y síntesis

$\checkmark$ Capacidad de investigación

$\checkmark$ Capacidad creativa

\section{Conclusión}


En todo proceso de cambio o reforma educativa, el docente es un componente nuclear a considerar, resulta utópico alcanzar una formación de alta calidad que propenda a la excelencia y pertinencia del Sistema de Educación Superior solo articulando dichas reformas a las necesidades de la transformación y participación social (fundamentales para alcanzar el Buen Vivir), sin tomar en cuenta especialmente al profesorado y sus competencias.

Los cambios que se están presentando en el entorno donde el docente universitario realiza su actividad profesional, en especial los introducidos por el Reglamento de Carrera y Escalafón del Profesor Investigador del Sistema de Educación Superior, el Reglamento de Régimen Académico y la nueva propuesta curricular que se aproxima, demandan nuevos planes en el proceso enseñanza aprendizaje, como lo menciona Tejada (2005) el cambio de paradigma educativo de un ejercicio enseñanza centrado en el profesor, a un ejercicio aprendizaje centrado en el estudiante.

Cambios estructurales como aquel que ubica a la carrera de Derecho dentro de las Ciencias Sociales y que otorga el título de Abogado de Los Tribunales y Juzgados (como es el caso de la Universidad Internacional del Ecuador), pasa a ser parte de una nomenclatura de títulos profesionales y grados académicos según el nivel de formación, es decir, campo amplio: Ciencias sociales, periodismo, información y derecho; campo específico: Derecho; campo detallado: Derecho; Titulación: Abogado/a.

Cambios en el diseño curricular, donde se revisan los objetivos de aprendizaje en términos de competencias y la evaluación por competencias; revisión de la metodología considerando las actividades de aprendizaje con docencia, de aplicación práctica y de trabajo autónomo que garanticen los resultados pedagógicos del tercer nivel de formación correspondiente al Abogado/a.

Cambios en el uso de los recursos de que dispone el docente, que requieren el dominio de herramientas de autoaprendizaje, un alto protagonismo de las bibliotecas virtuales, manejo de plataformas digitales para registro de actividades en aula, control de asistencia que permita verificar el cumplimiento de las actividades del profesor, etc.

La visión del docente con respecto a las competencias que debe adquirir el estudiante, responde al contexto, en este estudio se evidencia que en Europa la capacidad de generar nuevas ideas es un factor diferenciador, en términos de competencias, en los estudiantes de educación superior; por otro lado, en Latinoamérica en general, el compromiso ético y compromiso con la calidad son más importantes; en particular para el grupo de Derecho del proyecto Tuning la planificación, organización, comunicación, investigación y compromiso ciudadano es prioritario.

En el caso ecuatoriano, los docentes de Derecho, mantienen como prioridad las competencias genéricas instrumentales que están asociadas a la parte cognitiva, dejando de lado las competencias interpersonales y las competencias sistémicas. Esto nos permite tener una visión de la calidad de la docencia y del propio profesor como aspectos claves en todo el proceso de cambio que vive la educación superior. 
Por lo que la prioridad de la formación del personal docente e investigador, permitirá la polivalencia y flexibilidad que se requiere del docente, siendo la universidad corresponsable de propiciar este cambio, potenciar la preparación del docente y motivar la función investigadora con méritos y promoción profesional.

Contar con una propuesta de perfil competencial, contribuye a la identificación de las competencias necesarias para el desarrollo de la función de docente de la carrera de Derecho y, por consiguiente, detectar las necesidades de capacitación concretas comparando el dominio competencial actual y el requerido para un desarrollo óptimo de la función docente en la universidad.

Los planes formativos específicos que acerquen al docente a su realidad, que le permita comprender para transferir y el dominio de las competencias, como lo menciona Mas-Torelló y Olmos-Rueda (2016), son elementos que pueden incorporarse a lo que denominan los autores un correcto sistema de evaluación de los aprendizajes adquiridos y competencias desarrolladas por el docente en las tareas formativas y en el desarrollo de las funciones profesionales.

En contradicción con dos indicadores del modelo de evaluación de la carrera de Derecho, el primero la afinidad formación docente, que evalúa la proporción ponderada de las asignaturas impartidas por el docente cuyo título de cuarto nivel es afín a las asignaturas dictadas; y el segundo, la evaluación docente que, considerada la autoevaluación, evaluación por pares, evaluación por directivos, y evaluación por estudiantes.

El marco normativo de Educación Superior se centra en el ser humano y garantiza su desarrollo holístico, otorgando el protagonismo al estudiante, esto implica un cambio de mentalidad en la docencia universitaria que a base de reglamentos difícilmente podrá conseguirse, pues la norma es tan solo uno de los componentes del cambio social.

Por ello, resulta imprescindible una participación comprometida del docente y el trabajo en equipo, sin estas competencias será complicado avanzar hacia otro modelo de universidad de acuerdo al momento histórico que vive la educación superior ecuatoriana. Para adquirir este necesario compromiso, el docente demanda nuevas competencias profesionales que le forme y sensibilice hacia el reto que el Buen Vivir plantea; para ello la política universitaria tendrá que proporcionar medios y ofrecer estímulos hacia la coordinación y el trabajo en equipo. sobre:

Por otro lado, la organización personal del trabajo del docente, nos convoca cuestionarnos

$\checkmark \quad$ ¿Qué modelo de desarrollo académico se busca impulsar?

$\checkmark$ ¿Cómo combinar las actividades que desarrollan los docentes como la academia, investigación, vinculación con la comunidad y la gestión al modelo de desarrollo académico?

$\checkmark$ ¿Cómo, con qué tiempo, requisitos y recursos se debe desarrollar este distributivo docente? 
$\checkmark \quad$ ¿Es sostenible un escenario de calidad de la enseñanza - aprendizaje que se enmarca en una ingenua idea del desarrollo puntual de tareas del profesor, sin intervenir en otros ámbitos igual de complejos como los organizativos, institucionales, culturales, laborales y profesionales?

$\checkmark \quad$ ¿Es posible una educación por competencias con docentes de derecho alejados del libre ejercicio profesional por la carga laboral que modelo impone?

\section{Bibliografía}

Andrés Aucejo, E. (2009). Acerca de la'Revista de Educación y Derecho. Education and Law Review'. Revista de Educación y Derecho. Education and Law Review, 2009, p. 1121.Argudín, Y. (2009). Educación basada en competencias: nociones y antecedentes. (Primera). México: Trillas. Armijos, C. E. G., Barreno, F. L. L., \& Sánchez, F. de J. C. (2015). Los nuevos discursos, normativas y prácticas sobre educación superior en la República del Ecuador: Los énfasis en investigación e innovación. Avanzada Científica, 18(3), 15-31.Beneitone, P., Esquetini, C.,

González, J., Maletá, M. M., Siufi, G., \& Wagenaar, R. (2010). Informe Final Proyecto TuningAmérica Latina: reflexiones y perspectivas de la Educación Superior en América Latina 2004-2007. 2007. Bilbao: Universidad Deusto. Bilbao: Publicaciones de la Universidad de Deusto.Blas

Aritio, F. de A. (2014). Competencias profesionales en la formación profesional (Larousse-Alianza Editorial). Recuperado de http://www.mdconsult.internacional.edu.ec:2071/lib/bibliovirtualuidesp/detail.action?doc ID=11028703CES. Reglamento de Régimen Académico, RPC-SE-13-No.051·2013 Consejo de Educación Superior § Disposición general cuarta (2013).CES. (2017, Julio). Reglamento de Carrera y Escalafón del profesor e investigador del Sistema de Educación Superior.

INNOVA Research Journal 2018, Vol 3, No. 11, 119-138.Revistamensualde la UIDE extensiónGuayaquil136Cid, J. E., Cuadra, L. P., Cuevas, S. H., \& Villalobos, A. A. (2017).

Articulación Educación y Trabajo: Un estudio desde la Mirada de los Docentes de la Educación Técnica Regional Chilena y sus Necesidades de Perfeccionamiento Pedagógico.

Información tecnológica, 28(1), 25-34.Coraggio, J. L. (2005). Sobre la sostenibilidad de los emprendimientos mercantiles de la economía social y solidaria. Trabajo presentado en el Panel sobre "Nuevas formas asociativas para la producción", dentro del Seminario "De la Universidad pública a la sociedad argentina. El Plan Fénix en vísperas del segundo centenario. Una estrategia nacional de desarrollo con equidad." Universidad de Buenos Aires, 2-5. Recuperado de http://dhl.hegoa.info/ficheros/0000/0069/sostenibilidad_emprendimientos_mercantiles.pd fDe 
Vivero, F. (2014). La enseñanza del derecho encaminada a formar abogados. REDU. Revista de Docencia Universitaria, 12(3), 111-125.Delgado, G. L., García, C. F. J., \& Meza, S. E. (2010). Procedimiento para desarrollar la formación basada en competencias en la Educación Superior. En: Memorias del programa científico Universidad 2010. (Editorial Universitaria). Cuba. Recuperado de http: //www.ebrary.comDíaz, M. F. I. (2017). Perfil diferencial educativo. Análisis de la Realidad Nacional, 114(114).Espinosa, C. E. F., \& Aguilar, C. E. V. (2017). Desarrollo de habilidades investigativas en la educación superior. Académico, 4(1), 1-2.Fernández, L. R.,

González, L. D., \& Román, E. G. L. (2016). Competencias genéricas en docentes del nivel superior en el Estado de Guerrero/Generic competence on teaching staff of higher education in the State of Guerrero. RIDE Revista Iberoamericana para la Investigación y el Desarrollo Educativo, 6(12), 362-378.González, J., \& Wagenaar, R. (2006). Tuning Educational Structures in Eutope II. La contribución de las universidades al Proceso de Bolonia. Universidad de Deusto. Recuperado de http://www.unideusto.org/tuningeu/images/stories/Publications/Tuning_2_CAST_PR2_p df.pdfH

Ernández Sampieri, R., Fernández Collado, C., \& Baptista Lucio, P. (2014). Metodología de la investigación. Sexta Edición. Editorial Mc Graw Hill. México. 2014• Hernández, R. Metodología de la Investigación. 6a Edición, Mc Graw Hill, México.Jiménez-Vivas, A. (2009). Reflexiones sobre la necesidad de acercamiento entre universidad y mercado laboral. Revista Iberoamericana de Educación, 50(1), 1-8.

INNOVA Research Journal 2018, Vol 3, No. 11, 119-138.Revistamensualde la UIDE extensiónGuayaquil137López Gómez, E. (2016). En torno al concepto de competencia: un análisis de fuentes. Profesorado. Revista de Currículum y Formación de Profesorado, 20(1).Mas Torelló, Ó. (2011). El profesor universitario: sus competencias y formación. Recuperado de http://digibug.ugr.es/handle/10481/23166Mas Torelló, Ó., \& Tejada Fernández, J. (2013). Funciones y competencias en la docencia universitaria. Ed. Síntesis.Mas-Torelló, Ó., \& Olmos-

Rueda, P. (2016). El profesor universitario en el Espacio Europeo de Educación Superior: la autopercepción de sus competencias docentes actuales y orientaciones para su formación pedagógica. Revista mexicana de investigación educativa, 21(69), 437-470.Mulder, M., Weigel, T., \& Collins, K. (2007). The concept of competence in the development of vocational education and training in selected EU member states: a critical analysis. Journal of Vocational Education \& Training, 59(1), 67-88. Recuperado de http://www.tandfonline.com/doi/abs/10.1080/13636820601145630Musse, L., Barbé, C.,

Barranco, G., Coloma, R., Delmas, G., Lapenta, E.,... Ventura, J. (2014). Tuning América Latina. Educación Superior en América Latina: reflexiones y perspectivas en Derecho. Bilbao: Universidad de Deusto. Presidencia de la República. Reglamento General de la Ley Orgánica de Educación Superior, Registro Oficial Suplemento 526 Decreto Ejecutivo 865 $\S$ (2011).Rama Vitale, C. (2006). La tercera reforma de la educación superior en América 
Latina y el Caribe: masificación, regulaciones e internacionalización. Revista Educación y Pedagogía, Universidad de Antioquia. Facultad de Educación, XVIII (46), 11-24. Recuperado http://www.ub.edu/histodidactica/images/documentos/pdf/ESuperior\%20en\%20AMARI CA\%20

LATINA.pdf Ramos, P. (2016). Abogado y sociedad: deberes como profesional e implicación social. Registro Oficial. Ley Orgánica de Educación Superior, Pub. L. No. Ley \# 0, Suplemento 298 T. 4454-SNJ-10-1512 (2010).Rodríguez, A., \& Parietti, L. A. (2007). Educación-Trabajo ¿un vínculo en Estado de dependencia? Aproximaciones sobre la relación: Sistema Educativo -Sistema Productivo -Política de Gobierno. ?? De la escuela al trabajo?: la educación y el futuro laboral de los jóvenes en tiempos de globalización, 223. Recuperado de https://books.google.es/books?hl=es\&lr=\&id=UoB9mFHttQwC\&oi=fnd\&pg=PA223\&dq $=$ Educaci\%C3\%B3n-Trabajo+\%22estado+de+dependencia\%22\&ots=7GJnDEaU

INNOVA Research Journal 2018, Vol. 3, No. 11, 119-138.Revistamensualde la UIDE extensiónGuayaquil138rZ\&sig=zwrSZz8RXTRGuizNtmgcQZJ-TdURomero-Sandoval, A. R., \&

León Guajardo, M. (2017). Análisis documental del perfil competencial del docente universitario en un país latinoamericano de ingresos medios, mediante teoría fundamentada. CIAIQ 2017

Sáez López, J. M. (2017). Investigación educativa: fundamentos teóricos, procesos y elementos prácticos: enfoque práctico con ejemplos, esencial para TFG, TFM y tesis. Madrid: UNED -Universidad Nacional de Educación a Distancia. Recuperado de https: //www.mdconsult.internacional.edu.ec:2095Salgado, J. A. G. (2015). Problemas de precisión del discurs jurídic (aproximación des de l'àmbit de l'assessoria lingüística). Revista de Llengua i Dret, (64), 47-62.Sánchez Torelló, J. L. (2014).

Las competencias profesionales en el ejercicio de la abogacía.Sousa Santos, B. de. (2005). La universidad en el siglo XXI para una reforma democrática y emancipadora de la universidad. Recuperado de http://iep.udea.edu.co:8180/jspui/handle/123456789/267Tejada Fernández, J. (2002). El docente universitario ante los nuevos escenarios: implicaciones para la innovación docente. Acción pedagógica, 11(2), 30-42. Recuperado de http://www.saber.ula.ve/handle/123456789/17162Tejada Fernández, J. (2009).

Profesionalización docente en el escenario de la Europa de 2010. Una mirada desde la formación. Revista de educación, (349), 0463-477. Recuperado de http://ddd.uab.cat/record/127888/Tejada Fernández, J. (2012). La alternancia de contextos para la adquisición de competencias profesionales en escenarios complementarios de educación superior. En Educación XXI (Vol. 15, pp. 0017-40). Recuperado de http://ddd.uab.cat/record/123709/UNESCO. (2016). Estrategia de la UNESCO para la Enseñanza y Formación Técnica y Profesional (EFTP) (2016-2021). 
UNESCO.UNESCO. (2017). La educación al servicio de los pueblos y el planeta: creación de futuros sostenibles para todos (Primera). Paris: UNESCO. Zambrano, A. C. (2017). La relación entre conocimiento común y conocimiento científico en el contexto de la enseñanza, aprendizaje y cambio conceptual de las ciencias. TED: Tecné, Episteme y Didaxis, (3). 